\title{
TRATAMENTO SILVICULTURAL E INCREMENTO DIAMÉTRICO DE Platonia insignis Mart. (CLUSIACEAE) "BACURIZEIRO" EM DUAS FLORESTAS SECUNDÁRIAS EM BRAGANÇA, PA, BRASIL ${ }^{1}$
}

\author{
Rivaldo Costa Cardoso Junior ${ }^{2}$, Maria do Socorro Gonçalves Ferreira ${ }^{3}$, Fernando Cristovam da Silva \\ Jardim $^{4}$, Manoela Ferreira Fernandes da Silva ${ }^{5}$ Lucicléia Nascimento Esquerdo ${ }^{6}$
}

\begin{abstract}
RESUMO - Verificou-se neste trabalho o efeito de tratamentos silviculturais no incremento diamétrico de Platonia insignis, em duas florestas secundárias, com dois anos de acompanhamento (2005/2007). As florestas foram denominadas Unidades Agrárias (UA), onde foram instaladas quatro parcelas de 70 m x 105 m (UA1) e 35 m x 100 m (UA2). Dois tratamentos foram aplicados com duas repetições: T0 nenhuma intervenção; e T1 tratamento silvicultural, que consistiu de desbaste por abate e desbaste por anelamento, coroamento de $2 \mathrm{~m}$ e corte de cipós. Foram medidos todos os indivíduos de $P$. insignis com DAP $\geq 10 \mathrm{~cm}$. Foram utilizados os testes t, de Student, e de Mann Whitney, para comparação das médias. O incremento anual médio em diâmetro na T1 foi significativamente maior em ambas as florestas secundárias. UA1 apresentou $0,14 \mathrm{~cm}_{\text {ano }}{ }^{-1}$ para T0 e 0,25 cm.ano ${ }^{-1}$ para T1. Na UA2, a média foi de $0,53 \mathrm{~cm} \cdot \mathrm{ano}^{-1}$ para T0 e 0,93 cm.ano ${ }^{-1}$ para T1. Portanto, o efeito positivo dos tratamentos silviculturais no aumento diamétrico pode servir como subsídio para atividades de manejo sustentável e aplicação em florestas secundárias como indutor de crescimento.
\end{abstract}

Palavras-chave: Desbaste; Anelamento; Manejo sustentável.

\section{SILVICULTURAL TREATMENT AND DIAMETER INCREMENT OF Platonia insignis Mart. (CLUSIACEAE) "BACURIZEIRO" ON TWO SECONDARY FORESTS ON BRAGANÇA, PA, BRAZIL}

\begin{abstract}
The objective of this paper was to describe the effect of silvicultural treatments on diameter increment of Platonia insignis, in two secondary forests, for two years of monitoring (2005/2007). Each forest was denominated of Agrarian Unit (AU) where four plots were installed with $70 \mathrm{~m} \times 105 \mathrm{~m}$ (AU1) and 35 $m \times 100 m$ (AU2). Two treatments were applied with two replications, T0 - no intervention; and T1 - silvicultural treatment that consisted of thinning by felling and girdling, crowning of $2 \mathrm{~m}$ and cutting of lianas. All $\boldsymbol{P}$. insignis individuals were measured with $D B H \geq 10 \mathrm{~cm}$. To compare the means, Student test and Mann Whitney test were used. The average annual increment in diameter in T1 was significantly higher in both secondary forests. AU1 presented $0.14 \mathrm{~cm}$.year ${ }^{-1}$ for TO and $0.25 \mathrm{~cm}_{\text {.year }}{ }^{-1}$ for T1. In AU2, the average was $0.53 \mathrm{~cm} . y e a r^{-1}$ for TO and $0.93 \mathrm{~cm}$.year-1 for T1. Therefore, the positive effect of silvicultural treatments in the diameter increase can serve as subsidy for activities of sustainable management and application in secondary forests as growth inductor.
\end{abstract}

Keywords: Thinning; Girdling; Sustainable management.

\footnotetext{
${ }^{1}$ Recebido em 07.10.2009 aceito para publicação em 02.07.2014.

${ }^{2}$ Secretaria de Estado de Meio Ambiente, Pará, Brasil. E-mail: <rivasforestry@yahoo.com.br>.

${ }^{3}$ Empresa Brasileira de Pesquisa Agropecuária, Centro de Pesquisa Agroflorestal da Amazônia Ocidental, Brasil. E-mail: <socorrofer@gmail.com>.

${ }^{4}$ Instituto de Ciências Agrárias, Universidade Federal Rural da Amazônia, Brasil. E-mail: <fernando.jardim@ufra.edu.br>.

${ }^{5}$ Coordenação de Botânica, Museu Paraense Emílio Goeldi, Brasil. E-mail: <manoela@museu-goeldi.br>.

${ }^{6}$ Programa de Pós-Graduação em Botânica Tropical, Coordenação de Botânica, Museu Paraense Emílio Goeldi, Brasil. E-mail:<esquerdoflorestal@yahoo.com.br>.
} 


\section{INTRODUÇÃO}

As florestas secundárias são definidas como vegetações arbóreas que surgem após a remoção da vegetação original por consequência de distúrbios antrópicos ou naturais (BROWN; LUGO, 1990; CORLETT, 1994; PIRES-O’BRIEN; O’BRIEN, 1995; SMITH et al., 2000).

As florestas secundárias são caracterizadas por estarem em processo de desenvolvimento e passarem por diversas fases sucessionais até alcançarem a fase de estabilização. Dependendo do estágio sucessional, esse tipo de vegetação apresenta diversidade de espécies vegetais que podem oferecer serviços e produtos diversos.

Entre os vários recursos naturais que as florestas secundárias podem oferecer, a Platonia insignis é de grande importância, com destaque para a madeira e o fruto. Na região Bragantina, é comum encontrar densidades elevadas dessa espécie, que localmente são conhecidas como bacurizais.

A Platonia insignis apresenta porte arbóreo, com cerca de 15 a 35 m de altura, podendo atingir até 150 cm de diâmetro (CAVALCANTE, 1996; SHANLEY; MEDINA, 2005; HOMMA et al., 2007a). É planta perene que ocorre em baixa densidade na floresta primária, com característica marcante de se propagar vegetativamente pelo brotamento de raízes, que só se verifica quando clareiras são abertas, haja vista que a emissão dessas brotações só ocorre na presença de certo nível de luminosidade (CARVALHO; MÜLLER, 2007; HOMMA et al., 2007b). Apresenta tronco reto, com látex amarelo e galhos opostos em posição de V aberto, com folhas opostas e brilhosas (SHANLEY; MEDINA, 2005). A flor é hermafrodita e o fruto, do tipo baga (MOURÃO; BELTRATI, 1995; BATISTA; JARDIM, 2006).

O manejo através da aplicação de tratamentos silviculturais pode estimular o crescimento diamétrico de indivíduos arbóreos competidores em florestas secundárias. Segundo Ferreira et al. (1998), o crescimento pode ser definido como a relação de um tamanho especificado (DAP, altura etc.) entre dois inventários sucessivos de um povoamento florestal.

De acordo com Lamprecht (1990), Costa et al. (2001), Silva (2001) e Souza e Souza (2005), os tratamentos silviculturais aumentam significativamente o crescimento das árvores em florestas tropicais, podendo esse crescimento ser duplicado em relação ao que ocorre em uma floresta explorada e sem tratamento ou até ser quatro vezes maior em uma floresta sem distúrbios. Além disso, a aplicação desse recurso reduz a competição entre árvores por espaço, luz e nutrientes proporcionando aumento da sobrevivência e do crescimento - e o estabelecimento da regeneração natural das espécies de valor econômico, melhorando a qualidade e produtividade das florestas.

Nesse sentido, esta pesquisa teve como objetivo verificar o efeito de tratamentos silviculturais no incremento diamétrico de Platonia insignis.

\section{MATERIAL E MÉTODOS}

As áreas experimentais, de propriedade de pequenos produtores, foram denominadas Unidades Agrárias (UA). A UA1 possui aproximadamente 40 anos de idade, com área amostral de 2,96 ha, localizada na comunidade Benjamim Constant entre $01^{\circ} 11^{\prime} 18^{\prime \prime}$ latitude Sul e $46^{\circ} 40^{\prime} 38^{\prime \prime}$ de longitude Oeste. A UA2 tem cerca de 30 anos de idade, com área amostral de 1,4 ha, situada na comunidade de Enfarrusca entre $01^{\circ} 07^{\prime}$ ' 25" latitude Sul e $46^{\circ} 42^{\prime}$ ' de longitude Oeste. Ambas as comunidades pertencem ao Município de Bragança, Pará.

A vegetação da área é classificada como floresta ombrófila densa secundária (COORDENAÇÃO..., 2008). O clima da região éAw, de acordo com Köppen (ROCQUE, 1982). A temperatura média anual é de $25^{\circ} \mathrm{C}$ a $26^{\circ} \mathrm{C}$. A precipitação anual varia em torno de $2.200 \mathrm{~mm}$ a 3.000 mm (RIOS et al., 2002). A área está situada em uma zona de planície levemente ondulada, formada por sedimentos recentes, com declive máximo de 26 m (ROCQUE, 1982). O solo na região é predominantemente o Latossolo Amarelo Distrófico (LAD), textura média, caracterizado como profundo e de evolução avançada (EMBRAPA, 1999).

O experimento foi instalado em delineamento inteiramente ao acaso, com dois tratamentos e duas repetições no ano de 2005, assim especificados: TO - testemunha (sem intervenção); e T1 - aplicação de tratamentos silviculturais.

Cada parcela possui área de 70 m x 105 m na UA1; e $35 \mathrm{~m}$ x $100 \mathrm{~m}$ na UA2, deixando-se uma bordadura de 10 m entre elas (Figura 1). 

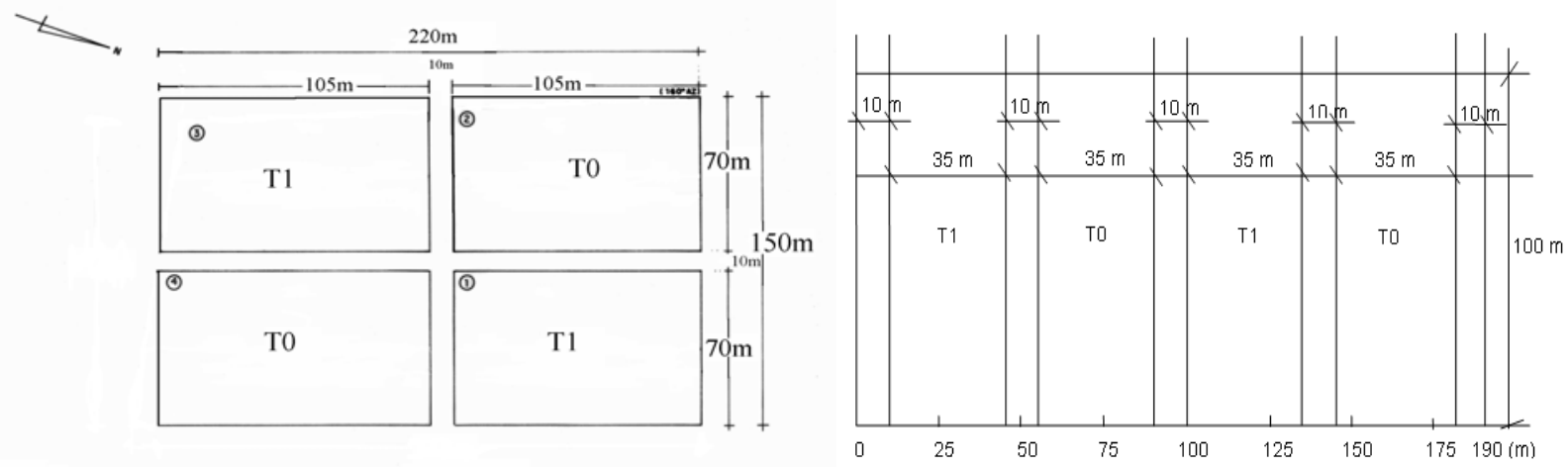

Figura 1 - Croquis da área de tratamento silvicultural Unidades Agrárias 1 e 2, em Bragança, Pará. $T_{0}=$ testemunha - nenhuma intervenção; e $T_{1}=$ parcelas onde foram aplicados os tratamentos silviculturais.

Figure 1 - Sketches of the silvicultural treatment area Agrarian Unit 1 and 2, in Bragança, Pará. $T_{0}=$ control - no intervention; and $T_{1}=$ parcels where silvicultural treatments were applied.

Antes da aplicação dos tratamentos, todos os indivíduos de Platonia insignis com Diâmetro a Altura do Peito (DAP) $\geq 10 \mathrm{~cm}$ foram identificados, medidos, numerados e etiquetados. Os indivíduos de $P$. insignis selecionados possuíam as seguintes características: Fuste completo (sem danos e podridão) e copa saudável com bom desenvolvimento (folhas visualmente verdes e vistosas e ramos bem distribuídos).

Os tratamentos silviculturais foram os seguintes: desbaste por abate (OLIVEIRA, 2005); desbaste por anelamento (DUBOIS, 1976); coroamento através da limpeza de ervas, plântulas e varas (indivíduos com $\mathrm{DAP} \geq 2,5 \mathrm{~cm}$ e menor que $5,0 \mathrm{~cm}$ ), num raio de $2 \mathrm{~m}$ em relação ao tronco do indivíduo selecionado; e corte de cipós (VIDAL et al., 2003).

Essas técnicas tiveram o propósito de eliminar indivíduos que competiam diretamente com os exemplares de $P$. insignis selecionados para o manejo.

O desbaste por abate foi realizado em espécimes com diâmetro à altura do peito (DAP) até $10 \mathrm{~cm}$, que competiam em nível de copa em um raio de até $20 \mathrm{~m}$. $\mathrm{O}$ anelamento foi realizado em indivíduos com DAP $>10 \mathrm{~cm}$, com o mesmo princípio do desbaste anterior.

O anelamento de espécimes de maior diâmetro teve como objetivo evitar danos à vegetação remanescente, com abertura indesejável ao dossel, ocasionada pela queda da árvore caso fosse derrubada. Segundo Lamprecht (1990), o anelamento faz que a árvore morra em pé lentamente, perdendo folhas e galhos e diminuindo os danos à vegetação remanescente.
O anelamento foi do tipo completo, de acordo com Sandel e Carvalho (2000), que consistiu na retirada da casca e do câmbio da árvore, formando um anel de aproximadamente $30 \mathrm{~cm}$ de largura, à altura de 1 $\mathrm{m}$ do solo.

Em 2007, foi feita nova medição dos indivíduos marcados de $P$. insignis preexistentes no período.

Foram realizadas análises de incremento no período de 2005 a 2007. O incremento em diâmetro foi calculado de acordo com a equação a seguir:

$$
I M A_{D A P}=D A P f-D A P i / t
$$

em que IMA ${ }_{\mathrm{DAP}}=$ incremento médio anual em DAP; DAPf = diâmetro à altura do peito final; DAPi = diâmetro à altura do peito inicial; e $\mathrm{t}$ = período de monitoramento (em anos).

Para o estudo da variação das médias de incremento médio anual entre os tratamentos, foram utilizados os testes de Mann-Whitney (U) e T, de Student, para comparação entre os valores de IMA, com o auxílio do software estatístico BIOSTAT 5.0. Na UA1, foi utilizado o teste U, devido ao fato de não apresentar distribuição normal dos dados. Na UA2, por apresentar distribuição normal, foi decidida a aplicação do teste T.

Foi analisada a distribuição diamétrica nas Unidades Agrárias 1 e 2, através da construção de gráficos por classe de diâmetro. Os gráficos foram realizados utilizando o programa MICROSOFT EXCEL. As classes de diâmetro ficaram distribuídas em: Classe de DAP 1 (C1), no intervalo de DAP (cm) de 10 - 20; C2 de 20,1 - 30; C3 de 30,1 - 40; C4 de 40,1 - 50; C5 de 50,1 - 60; e C6 > 60.

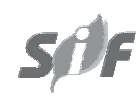

Revista Árvore, Viçosa-MG, v.38, n.5, p.889-898, 2014 


\section{RESULTADOS}

Foram identificados e marcados 352 indivíduos de Platonia insignis em 2005, com DAP $\geq 10 \mathrm{~cm}$. Nas parcelas-testemunha (T0), foram registrados 185 indivíduos, distribuídos em 119 para UA1 e 66 para UA2. Nas parcelas com aplicação de tratamento silvicultural (T1), foram registrados 167 indivíduos, distribuídos em 92 para UA1 e 75 para UA2.

Em 2007, esse número diminuiu para um total de 341 indivíduos em decorrência da mortalidade nas áreas de estudo, com 176 indivíduos nas parcelas T0, distribuídos em 111 para UA1 e 65 para UA2. Nas parcelas submetidas a tratamento silvicultural, foram catalogadas 165 árvores de $P$. insignis, distribuídas em 91 para UA1 e 74 para UA2. Foram identificados e eliminados 397 indivíduos competidores (Tabela 1), que representaram, em média, 1,7 árvore desbastada para cada exemplar de $P$. insignis beneficiado na UA1 e 3,2 árvores desbastadas de cada espécime beneficiado na UA2. O número de indivíduos anelados foi maior na UA1, onde a vegetação era mais desenvolvida, por ser mais antiga que a UA2, o que justificou o uso da técnica.

Na Tabela 2, relacionam-se as espécies desbastadas no experimento. As árvores com maior número de indivíduos desbastados foram Platonia insignis, Neea guianensis, Tapirira guianensis e Maprounea guianensis, com 30, 25, 11 e 10 indivíduos, respectivamente, na UA1. Na UA2, foram Platonia insignis, Croton matourensis e Himatanthus sucuuba, com 135, 23 e 9 indivíduos desbastados, respectivamente. Estima-se, portanto, que a abundância da espécie $P$. insignis foi representativa nas comunidades estudadas.

O incremento médio anual em diâmetro nas parcelas com aplicação de tratamentos silviculturais foi significativamente maior do que a encontrada nas parcelas-testemunha, em ambas as florestas secundárias em estudo, considerando-se os dois anos do período de acompanhamento (Mann-Whitney para a UA1 com $\mathrm{p}<0.0001$; teste $\mathrm{T}$ para a UA2 com $\mathrm{p}<0,0001$; $\alpha=$ $0,05)$.

Os incrementos médios anuais encontrados entre os tratamentos nas Unidades Agrárias foram de 0,14 cm.ano ${ }^{-1}$ no tratamento-testemunha (T0) e 0,25 cm.ano ${ }^{-1}$, com aplicação de tratamento silvicultural (T1), na UA1.

Na UA2, os incrementos médios anuais foram de $0,53 \mathrm{~cm}$. ano ${ }^{-1}$ no tratamento T0 e $0,93 \mathrm{~cm}$. ano ${ }^{-1}$ no tratamento $\mathrm{T} 1$.

As diferenças encontradas entre as médias de incremento diamétrico do tratamento-testemunha em relação àquele com tratamento silvicultural na UA1 e UA2 estão representadas na Figura 2.

Observou-se distribuição decrescente na forma de J-invertido na UA1, ou seja, maior número de indivíduos nas classes inferiores de diâmetro e menor número de indivíduos nas classes superiores, tanto no ano 2005 quanto no ano 2007.

Observou-se que o número de indivíduos no tratamento T1, no ano 2005, foi maior em relação aos resultados obtidos no ano 2007. Esse número ficou acima de 70 indivíduos na Classe de diâmetro 1 (C1) em 2005, enquanto em 2007 esse número foi reduzido, ficando abaixo de 70 indivíduos. Esse resultado, provavelmente, foi devido ao incremento diamétrico, o que provocou a mudança de classe de tamanho (Figura 3).

No tratamento T0 da UA1, a diferença do número de indivíduos na C1, em 2005, foi muito pequena em relação ao ano 2007. Isso, provavelmente, foi devido ao fato de esse tratamento não ter sofrido intervenção silvicultural, o que não influenciou no incremento diamétrico, que consequentemente não provocou mudança de classe de diâmetro no povoamento.

Tabela 1 - Distribuição do número de indivíduos de $P$. insignis selecionados e competidores encontrados no período de dois anos, nas UA1 e UA2, em Bragança, Pará.

Table 1 - Distribution of the number of selected individuals of $\boldsymbol{P}$. insignis and competitors found in the period of two years in the AU1 and AU2, in Bragança, Pará.

\begin{tabular}{|c|c|c|c|c|c|c|c|}
\hline \multirow[t]{3}{*}{ Unidade Agrária } & \multicolumn{4}{|c|}{$\mathrm{N}^{\circ}$ de indivíduos selecionados } & \multicolumn{3}{|c|}{$\mathrm{N}^{\circ}$ de indivíduos eliminados } \\
\hline & \multicolumn{2}{|c|}{2005} & \multicolumn{2}{|c|}{2007} & \multirow[t]{2}{*}{ Corte } & \multirow[t]{2}{*}{ Anelamento } & \multirow[t]{2}{*}{ Total } \\
\hline & T 0 & $\mathrm{~T} 1$ & T0 & $\mathrm{T} 1$ & & & \\
\hline UA1 & 119 & 92 & 111 & 91 & 74 & 84 & 158 \\
\hline UA2 & 66 & 75 & 65 & 74 & 166 & 71 & 237 \\
\hline Total & 185 & 167 & 176 & 165 & 240 & 155 & 395 \\
\hline
\end{tabular}

Revista Árvore, Viçosa-MG, v.38, n.5, p.889-898, 2014 
Tabela 2 - Lista das espécies encontradas competindo com os exemplares selecionados de Platonia insignis e que foram desbastadas na UA1 e UA2, em Bragança, Pará.

Table 2 - List of species competing with Platonia insignis, selected by thinning and girdling in AU1 and AU2 in Bragança, Pará.

\begin{tabular}{|c|c|c|c|c|c|}
\hline \multicolumn{3}{|l|}{ Unidade Agrária 1} & \multicolumn{3}{|l|}{ Unidade Agrária 2} \\
\hline \multirow[t]{2}{*}{ Espécie } & \multicolumn{2}{|c|}{ Indivíduos desbastados } & \multirow[t]{2}{*}{ Espécie } & \multicolumn{2}{|c|}{$\overline{\text { Indivíduos desbastados }}$} \\
\hline & Abate & Anelamento & & Abate & Anelamento \\
\hline $\begin{array}{l}\text { Abarema jupunba (Willd.) Britton } \\
\text { \& Killip }\end{array}$ & 2 & 1 & $\begin{array}{l}\text { Abarema jupunba (Willd.) Britton } \\
\text { \& Killip }\end{array}$ & 2 & - \\
\hline Agonandra brasiliensis Miers ex Benth. & 1 & 2 & Andira retusa (Poir.) Kunth & - & 2 \\
\hline Alibertia sp. & 1 & - & Annona paludosa Aubl. & 2 & - \\
\hline Bombax paraense Ducke & 1 & - & Bombax globosum Aubl. & 1 & - \\
\hline Bowdichia nitida Spruce ex Benth. & - & 2 & Byrsonima aerugo Sagot & - & 2 \\
\hline Byrsonima aerugo Sagot & - & 1 & Byrsonima densa (Poir.) DC. & - & 1 \\
\hline Connarus sp. & 1 & 1 & Casearia decandra Jacq. & 2 & - \\
\hline $\begin{array}{l}\text { Couratari oblongifolia Ducke \& } \\
\text { R. Knuth }\end{array}$ & 1 & 1 & Clusia sp. & 1 & - \\
\hline Croton matourensis Aubl. & - & 4 & $\begin{array}{l}\text { Couratari oblongifolia Ducke \& } \\
\text { R. Knuth }\end{array}$ & 1 & - \\
\hline Dialium guianense (Aubl.) Sandwith & 1 & 1 & Croton matourensis Aubl. & 14 & 9 \\
\hline Eugenia lambertiana DC. & 3 & 3 & Dipteryx odorata (Aubl.) Wild. & 1 & - \\
\hline Eugenia tapacumensis O.Berg & - & 1 & $\begin{array}{l}\text { Eschweilera coriacea (DC.) } \\
\text { S.A. Mori }\end{array}$ & 5 & 1 \\
\hline Franchetella gongrijpii (Eyma) Aubrév. & 1 & - & Eugenia tapacumensis O.Berg & 3 & - \\
\hline Guatteria ovalifolia R.E. Fr. & 1 & - & Guatteria poeppigiana Mart. & 1 & - \\
\hline Gustavia angusta J.F.Gmel. & 4 & 2 & Guatteria sp. & 1 & - \\
\hline $\begin{array}{l}\text { Himatanthus sucuuba (Spruce ex } \\
\text { Müll. Arg.) Woodson }\end{array}$ & - & 3 & $\begin{array}{l}\text { Himatanthus sucuuba (Spruce ex } \\
\text { Müll. Arg.) Woodson }\end{array}$ & 7 & 2 \\
\hline Inga sp. & - & 1 & Inga heterophylla Willd. & 1 & - \\
\hline Licania lata J.F. Macbr. & 2 & 1 & Inga sp. & 2 & - \\
\hline Lacistema pubescens Mart. & 4 & 1 & Iryanthera juruensis Warb. & 1 & - \\
\hline Lecythis lurida (Miers) S.A Mori & - & 2 & Licania densiflora Kleinhoonte & 3 & - \\
\hline Lecythis usitata Miers & - & 1 & Maprounea guianensis Aubl. & 1 & 2 \\
\hline Maprounea guianensis Aubl. & 3 & 7 & Myrcia atramentifera Barb. Rodr. & 1 & - \\
\hline Myrcia bracteata (Rich.) DC. & 1 & - & Myrcia silvatica Barb. Rodr. & 3 & - \\
\hline Myrciaria tenella (DC.) O. Berg & 3 & 1 & Ocotea guianensis Aubl. & 1 & - \\
\hline Neea guianensis Ruiz \& Pav. & 20 & 5 & Ormosia paraensis Ducke & 3 & 3 \\
\hline Ocotea guianensis Aubl. & - & 1 & Ormosiopsis flava (Ducke) Ducke & 2 & 2 \\
\hline Ouratea castaneaefolia (DC.) Engl. & 1 & 1 & Platonia insignis Mart. & 92 & 43 \\
\hline Pilocarpus sp. & 2 & 2 & $\begin{array}{l}\text { Rheedia acuminata (Ruiz \& Pav.) } \\
\text { Planch. \& Triana }\end{array}$ & 2 & - \\
\hline Platonia insignis Mart. & 6 & 24 & Swartzia arborescens (Aubl.) Pittier & 1 & - \\
\hline $\begin{array}{l}\text { Pogonophora achomburgkiana } \\
\text { Miers ex Benth. }\end{array}$ & 2 & 2 & Tachigalia paniculata Ducke & - & 1 \\
\hline Sacoglottis amazonica Mart. & 5 & - & Talisia guianensis Aubl. & 1 & - \\
\hline Sclerolobium chrysophyllum Poepp. & 1 & 2 & Tapirira guianensis Aubl. & 1 & 1 \\
\hline Simarouba amara Aubl. & - & 1 & Xilopia frutescens Aubl. & - & 1 \\
\hline $\begin{array}{l}\text { Stryphnodendron guianensis } \\
\text { (Aubl.) Benth. }\end{array}$ & - & 1 & não identificadas & 10 & 1 \\
\hline Tapirira guianensis Aubl. & 4 & 7 & & & \\
\hline Talisia guianensis Aubl. & 1 & - & & & \\
\hline Thyrsodium paraense Huber & 2 & - & & & \\
\hline Tovomita brevistaminea Engl. & - & 1 & & & \\
\hline Trattinickia rhoifolia Willd. & - & 1 & & & \\
\hline
\end{tabular}

Na UA2, não houve indivíduos nas classes superiores, ou seja, com DAP $>30 \mathrm{~cm}$, no início e fim do experimento. Foi encontrada pequena diferença entre o número de indivíduos nas Classes C1 e C2, tanto no ano 2005 quanto em 2007, conforme pode ser observado na Figura 3.

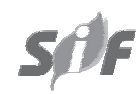

Revista Árvore, Viçosa-MG, v.38, n.5, p.889-898, 2014 

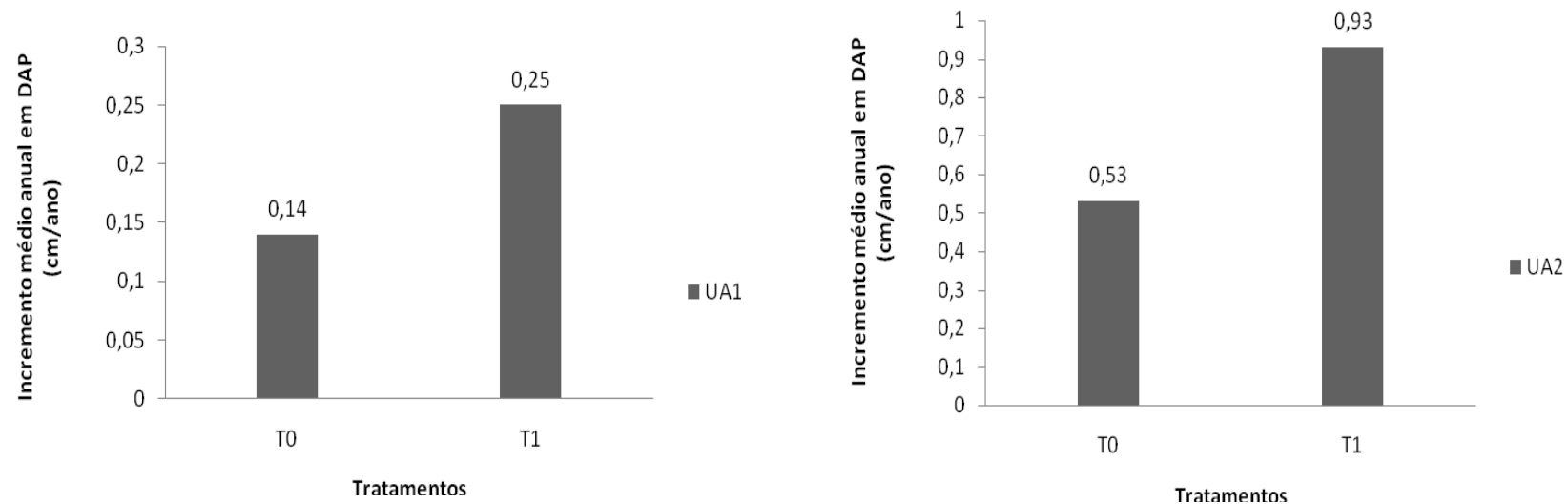

Figura 2 - Incremento médio anual em DAP de P. insignis, por tratamento, na UA1 (à direita) e UA2 (à esquerda), no período de 2005 a 2007, em Bragança, Pará.

Figure 2 - Mean annual increment in DBH of P. insignis, by treatment, in AU1 (on the right) and AU2 (on the left) in the period of 2005 to 2007, in Bragança, Pará.
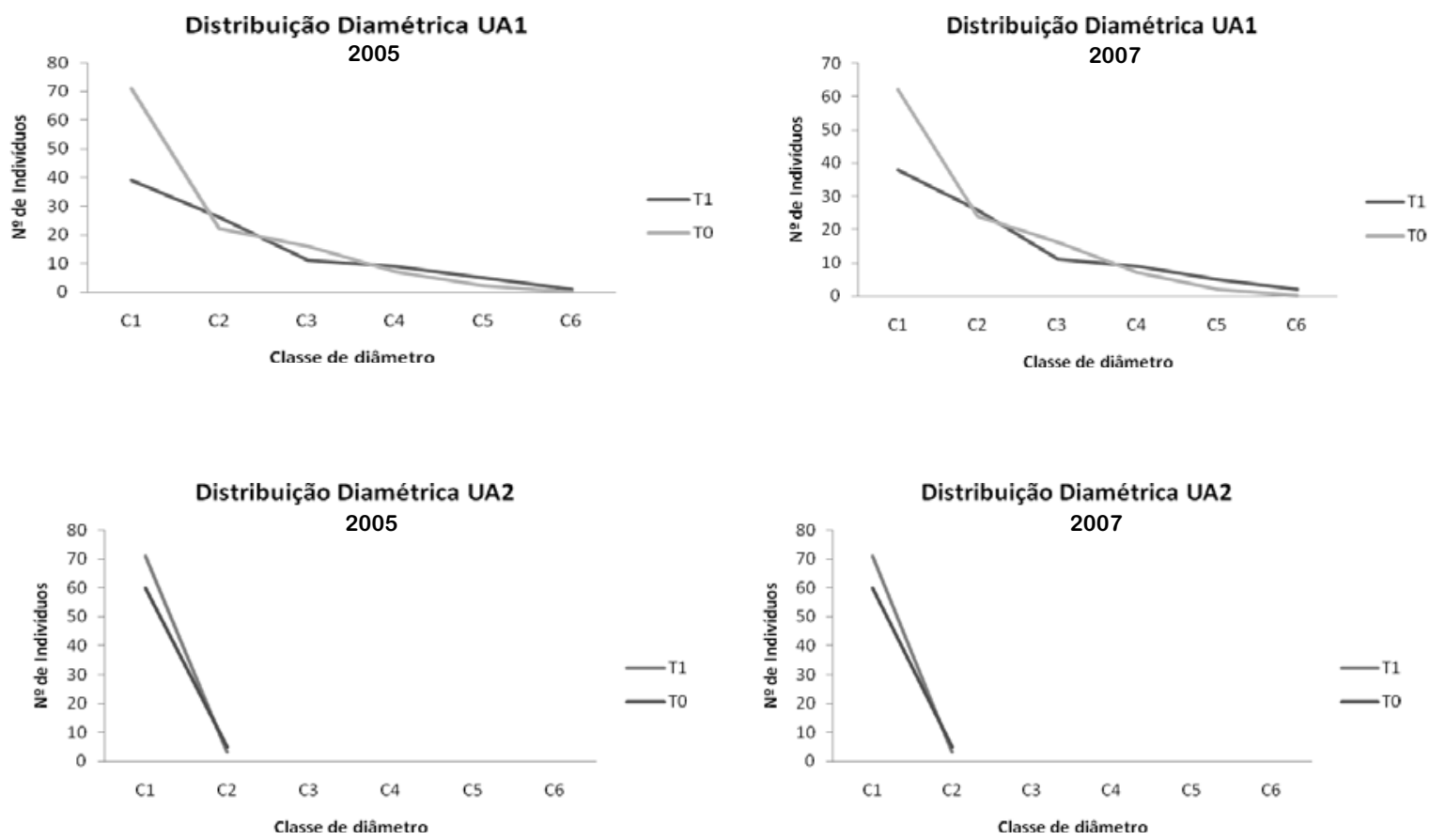

Figura 3 - Distribuição diamétrica de indivíduos de $P$. insiginis na UA1 e UA2 nos anos 2005 e 2007 com ( $\left.\mathrm{T}_{1}\right)$ e sem ( $\left.\mathrm{T}_{0}\right)$ na aplicação de tratamento silvicultural, Bragança, Pará.

Figure 3 - Diameter distribution of individuals of P. insiginis in AU1 and AU2 in 2005 and 2007 with $\left(T_{1}\right)$ and without $\left(T_{0}\right)$ in the application of silvicultural treatment, Bragança, Pará.

\section{DISCUSSÃO}

Guariguata (1999) comprovou que o crescimento diamétrico de quatro espécies, na Costa Rica, beneficiadas por desbaste foi altamente significativo
(Mann Whitney, p < 0,0001), com dois anos de acompanhamento do experimento. Ferreira (2008) constatou essa mesma tendência para beneficiamento de Platonia insignis com DAP $\geq 10 \mathrm{~cm}$, em Bragança, PA, Brasil.

Revista Árvore, Viçosa-MG, v.38, n.5, p.889-898, 2014 
Os resultados desse estudo de Ferreira (Op. cit.), quanto a incremento diamétrico em floresta secundária acima de 20 anos, foram semelhantes aos encontrados na UA2, nesta pesquisa, com $0,52 \mathrm{~cm}$ no T0 e 0,96 cm no T1. Diferentemente de Muniz et al. (2007), que em duas florestas secundárias, em Bragança, PA, encontraram médias de incremento diamétrico de 1,59 cm na floresta sem intervenção e 1,66 cm com a aplicação de tratamento silvicultural, com DAP $\geq 5 \mathrm{~cm}$. Ressaltase que na pesquisa de Muniz et al. (2007) foi aplicado tratamento silvicultural em várias espécies.

Em seus estudos, Guariguata (1999) e Ferreira (2008) sugeriram que os tratamentos silviculturais influenciam no incremento diamétrico de espécies demandantes de luz, com dois anos de monitoramento, como no caso deste estudo com $P$. insignis, o que corrobora a hipótese desta pesquisa.

Na pesquisa de Muniz et al. (2007), os tratamentos não foram significativos. Isso pode ser devido ao histórico de uso das áreas, pois, na Costa Rica, a floresta foi cortada para a criação de pasto e não houve queima da vegetação; no Nordeste do Pará houve corte seguido de queima para plantio de espécies agronômicas. Entretanto, o estudo de Ferreira (2008) ocorreu na mesma região do estudo de Muniz et al. (2007), com o mesmo histórico de uso. Várias causas podem ter influenciado nas diferenças de resultados desses estudos, como idade das florestas, intensidades dos tratamentos silviculturais e tipo solo, por exemplo.

Azevedo (2006) argumentou a relevância de se adotarem intervalos de dois anos e períodos longos para o estudo da dinâmica florestal. Entretanto, Guariguata (1999) verificou alto nível de significância em um ano de acompanhamento, com média de incremento diamétrico de $1,4 \mathrm{~cm}$ nas parcelas tratadas e $0,9 \mathrm{~cm}$ no grupo-controle.

O contrário foi observado no trabalho de Alvino et al. (2006), que aplicaram tratamentos silviculturais em florestas secundárias para beneficiamento de $P$. insignis com DAP $\geq 10 \mathrm{~cm}$. Esses autores não encontraram diferenças significativas entre os tratamentos. Para eles, a causa da não significância foi o curto período de monitoramento, no caso um ano. É válido ressaltar que esses tratamentos silviculturais foram de baixa intensidade, se comparados aos deste trabalho.
Os resultados altamente significativos verificados neste experimento podem ser, também, advindos da diminuição da competição por nutrientes, água e espaço para os indivíduos beneficiados pelos tratamentos silviculturais, associados a outros fatores anteriores aos tratamentos. Segundo Husch et al. (1982) e Lamprecht (1990), o crescimento diamétrico de árvores tropicais é influenciado pelas características da espécie, interagindo com condições ambientais, como fatores climáticos, pedológicos, topográficos e de competição.

Jardim et al. (2007), analisando o crescimento diamétrico em relação ao tamanho de clareiras de seis espécies na Amazônia oriental, verificaram que houve diferença significativa no incremento diamétrico ao comparar os tamanhos das clareiras, com dois anos de período de acompanhamento. De acordo com esses autores, o ambiente de clareira proporcionou condições microclimáticas às espécies, que foram favorecidas no incremento diamétrico.

D’Oliveira e Braz (2006), avaliando a dinâmica de uma floresta tropical semiperenifólia manejada no Estado do Acre, verificaram o incremento diamétrico de espécies quanto ao grau de iluminação após a exploração florestal, com DAP $\geq 20 \mathrm{~cm}$. Esses autores evidenciaram que a exposição das copas à luz do sol apresentou forte influência no incremento em diâmetro.

Mory e Jardim (2001) verificaram, em uma floresta madura na Amazônia ocidental brasileira, que a abertura de dossel, por anelamento, trouxe efeitos benéficos para a espécie Goupia glabra Aubl. (cupiúba) nos níveis de desbastes por anelagem mais drásticos (75\% e $100 \%$ de desbaste). Constataram crescimento diamétrico positivo de indivíduos com DAP $\geq 5 \mathrm{~cm}$.

De acordo com Silva et al. (1995), o efeito da exposição das copas à luz solar no que diz respeito ao incremento diamétrico de espécies tropicais tem forte correlação entre classe de iluminação de copa e crescimento.

Ainda que as espécies que Jardim et al. (2007) estudaram fossem plântulas em floresta primária e os estudos de D’Oliveira e Braz (2006), Mory e Jardim (2001) e Silva et al. (1995) tenham sido realizados também em florestas maduras, a abertura de dossel provocada pelos tratamentos silviculturais na floresta secundária possivelmente melhorou a condição microclimática dos indivíduos de $P$. insignis, ocasionando aumento

Revista Árvore, Viçosa-MG, v.38, n.5, p.889-898, 2014 
diamétrico. Essa tendência pode ser também observada nos trabalhos de Mesquita (2000) e Vidal et al. (2002) com outras espécies.

De acordo com os resultados, pode-se relatar que alguns indivíduos de $P$. insignis não tiveram aumento de diâmetro $(0,00 \mathrm{~cm})$ no período de acompanhamento na UA1. Esse fenômeno ocorreu somente no tratamento T0 da UA2, com número baixo de indivíduos sem incremento.

A maior ausência de incremento encontrada no tratamento T0 da UA1 pode ter sido provocada pela presença de cipós. Silva (2001) e Engel et al. (1998) argumentaram a importância de manejar florestas infestadas por cipós na Amazônia, uma vez que essas plantas prejudicam o crescimento diamétrico por competição.

Vidal et al. (2002), analisando uma floresta perenifólia em Paragominas e medindo todos os indivíduos com DAP $\geq 10 \mathrm{~cm}$, verificaram que árvores sem cipós apresentaram incremento diamétrico duas vezes maior que as árvores com cipós.

Foi observada na UA1 a sobrevivência de indivíduos ao anelamento. Essa sobrevivência interferiu no crescimento dos indivíduos de $P$. insignis selecionados para o manejo, apesar de não ter sido analisado esse aspecto (os indivíduos sobreviventes à anelagem).

Segundo Carvalho (1981), o anelamento elimina em média $21 \%$ dos indivíduos até um ano após a sua aplicação. No entanto, três anos após a operação a mortalidade sobe para $76 \%$. Este estudo foi monitorado durante dois anos, e é possível que fosse necessário um período mais longo de monitoramento para que o desbaste por anelamento tivesse maior efeito. Entretanto, Sandel et al. (1998) e Sandel e Carvalho (2000) evidenciaram que algumas espécies em floresta madura apresentaram resultados insatisfatórios com a aplicação de anelamento, em que a mais resistente só poderá ser eliminada mediante o uso de produto arboricida ou a derrubada.

Alvino et al. (2006), avaliando a sobrevivência à anelagem em floresta secundária, constataram 100\% nas espécies Platonia insignis Mart., Pogonophora schomburgkiana Miers ex Benth. e Ouratea castaneaefolia (DC.) Engl. e 40\%, 25\% e 20\% nas espécies Neea sp., Eschweilera coriacea (DC.) S. A. Mori e Maprounea guianensis Aubl., respectivamente. Da mesma maneira, interpreta-se que os resultados da UA2 foram superiores aos da UA1, porque houve mais indivíduos anelados, propiciando retardamento na liberação de copa dos exemplares selecionados de $P$. Insignis.

\section{CONCLUSÃO}

O manejo no decurso da aplicação de tratamentos silviculturais pode estimular o crescimento diamétrico de Platonia insignis em florestas secundárias.

Os tratamentos silviculturais influenciaram o incremento diamétrico nos indivíduos selecionados de $P$. insignis em ambas as UA.

Nesse sentido, o efeito positivo dos tratamentos silviculturais pode servir como subsídio para o planejamento de atividades de manejo florestal sustentável, principalmente para agricultores de base familiar, e contribuir para o desenvolvimento da floresta secundária.

\section{REFERÊNCIAS}

ALVINO, F. O.; RAYOL, B. P.; SILVA, M. F. F. Avaliação de tratamentos silviculturais aplicados a espécies competidoras de Platonia insignis Mart. (Clusiaceae), em floresta secundária na Zona Bragantina, Pará, Brasil. Revista de Ciências Agrárias, n.45, p.45-57, 2006.

AZEVEDO, C. P. Dinâmica de florestas submetidas a manejo na Amazônia Oriental: experimentação e simulação. 2006. 254f. Tese (Doutorado em Engenharia Florestal) Universidade Federal do Paraná, Curitiba, 2006.

BATISTA, F. J.; JARDIM, M. A. G. Notas sobre a morfologia floral e a fenologia do bacurizeiro (Platonia insignis Mart.) Clusiaceae, no município de Bragança, Estado do Pará. Boletim do Museu Paraense Emílio Goeldi, Ciências Naturais, v.1, n.1, p.183-186, 2006.

BROWN, S.; LUGO, A. E. Tropical secondary forests. Journal of Tropical Ecology, v.6, n.1, p.1-32, 1990.

CARVALHO, J. E. U.; MÜLLER, C. Propagação do bacurizeiro. In: LIMA, M. C. (Org). Bacuri: agrobiodiversidade. São Luís: Instituto Interamericano de Cooperação para a Agricultura, 2007. p.29-46. 
CARvalho, J. O. P. Anelagem de árvores indesejáveis em floresta tropical densa na Amazônia. Belém: Embrapa-CPATU, 1981. 11p. (Boletim de Pesquisa, 22).

CAVALCANTE, P. B. Frutas comestíveis da Amazônia. Belém: CNPq/MPEG, 1996. 279p.

COORDENAÇÃODE RECURSOS NATURAISE ESTUDOS AMBIENTAIS. IBGE, 2008. em < ftp:// geoftp.ibge.gov.br/mapas/tematicos/tematico_estadual/ pa_vegetacao.pdf $>$ acesso em: 17 de jun. de 2009.

CORLETT, R. T. What is secondary forests? Journal of Tropical Ecology, v.10, n.3, p.445-447, 1994.

COSTA, D. H. M.; SILVA, S. M. A.; SILVA, J. N. M. Efetividade e custos do desbaste com aplicação de arboricida em floresta natural na região do Tapajós, Pará e Jarí, Amapá. In: SILVA, J. N. M.; CARVALHO, J. O. P.; YARED, J. A. G. (Ed.). Silvicultura na Amazônia Oriental: contribuições do projeto Embrapa/ DFID. Belém: Embrapa Amazônia Oriental/DFID, 2001. p.339-352.

d'OLIVEIRA, M. V. N.; BRAZ, E. M. Estudo da dinâmica da floresta manejada no projeto de manejo florestal comunitário do PC Pedro Peixoto na Amazônia Ocidental. Acta Amazônica, v.36, n.2, p.177-182, 2006.

DUBOIS, J. C. L. Preliminary management forest guidelines for the National Forest of Tapajós. Belém: IBDF/PRODEPEF, 1976. 42p.

EMPRESA BRASILEIRA DE PESQUISA AGROPECUÁRIA - EMBRAPA. Centro Nacional de Pesquisa de Solo. Sistema brasileiro de classificação dos solos. Brasília: Embrapa, Produção de Informações, 1999. 412p.

ENGEL, V. L.; FONSECA, R. C. B.; OLIVEIRA, E. R. Ecologia de lianas e o manejo de fragmentos florestais. Série Técnica IPEF, v.12, n.32, p.43-64, 1998.

FERREIRA, M. S. G. Bacurizeiro (Platonia insignis Mart.) em florestas secundárias: possibilidades para o desenvolvimento sustentável no Nordeste Paraense. 2008. 231f. Tese (Doutorado em Desenvolvimento Sustentável) - Universidade de Brasília, Brasília, 2008.
FERREIRA, R. L. C.; SOUZA, A. L.; JESUS, R. M. Ingresso e mortalidade em uma floresta secundária de transição. Revista Árvore, v.22, n.2, p.155-162, 1998.

GUARIGUATA, M. R. Early response of selected tree species to liberation thinning in a young secondary forest in Northeastern Costa Rica. Forest Ecology and Management, v.124, p.255-261, 1999.

HOMMA, A. K. O. et al. Manejando a planta e o homem: os bacurizeiros do nordeste paraense e da ilha de Marajó. Amazônia: Ciência \& Desenvolvimento, v.2, n.4, p.119-135, 2007a.

HOMMA, A. K. O. et al. Manejando a planta e o homem: os bacurizeiros no Nordeste paraense. In: LIMA, M. C. (Org.). Bacuri: agrobiodiversidade. São Luís: Instituto Interamericano de Cooperação para a Agricultura, 2007b. p.171-210.

HUSCH, B.; MILLER, C. I.; BEERS, T. W. Forest mensuration. 3.ed. New York: John Wiley \& Sons, 1982. 402p.

JARDIM, F. C. S.; SERRÃO, D. R.; NEMER, T. C. Efeito de diferentes tamanhos de clareiras, sobre o crescimento e a mortalidade de espécies arbóreas, em Moju-PA. Acta Amazônica, v.37, n.1, p.37-484, 2007.

LAMPRECHT, H. Silvicultura nos Trópicos. Cooperação Técnica-RFA. Eschborn, 1990. 343p.

MESQUITA, R. C. G. Management of advanced regeneration in secondary forests of the Brazilian Amazon. Forest Ecology and

Management, v.130, p.131-140, 2000.

MOURÃO, K. S. M.; BELTRATI, C. M. Morfologia dos frutos, sementes e plântulas de Platonia insignis Mart. (Clusiaceae). II. Morfoanatomia dos frutos e sementes maduros. Acta Amazônica, v.25, n.1/2, p.33-46, 1995.

MORY, A. M.; JARDIM, F. C. S. Comportamento de Goupia glabra Aubl. (Cupiúba) em diferentes níveis de desbastes por anelamento em florestas naturais. Revista de Ciências Agrárias, n.36, p.55-66, 2001.

Revista Árvore, Viçosa-MG, v.38, n.5, p.889-898, 2014 
MUNIZ, A. L. V. et al. Dinâmica de floresta secundária com e sem tratamento silvicultural para fins de manejo no nordeste paraense.

Amazônia: Ciência \&

Desenvolvimento, v.2, n.4, p.53-65, 2007.

OLIVEIRA, L. C. Efeito da exploração da madeira e de diferentes intensidades de desbastes sobre a dinâmica da vegetação de uma área de 136 ha na Floresta Nacional do Tapajós. Tese (Doutorado em Recursos Florestais), Escola Superior de Agricultura "Luiz de Queiroz", Universidade de São Paulo, Piracicaba. 2005. 195 p.

PIRES O’ BRIEN, M. J.; O’ BRIEN, C. M. Ecologia e modelamento das florestas tropicais. Belém: FCAP, Serviço de Documentação e Informação, 1995. 400p.

RIOS, M.; CORDEIRO, M. R.; CONSTANT, C. B. Guia Etnobotânica de la Comunidad de Benjamin Constant Estado do Pará, Brasil. The field museum. Chicago: 2002. 150p.

ROCQUE, C. História dos Municípios do Estado do Pará. Belém: 1982. v.1. 279p.

SANDEL, M. P.; CARVALHO, J. O. P. Anelagem de árvores como tratamentos silviculturais em florestas naturais da Amazônia brasileira. Revista de Ciências Agrárias, n.33, p.9-32, 2000.

SANDEL, M. P.; BAIMA, A. M. V.; CARVALHO, J. $O$. $P$. Teste de anelagem em espécies arbóreas na floresta Amazônica. Belém: Embrapa-CPATU, 1998. 13p. (Embrapa-CPATU. Circular técnico, 75).
SHANLEY, P.; MEDINA, G. Frutíferas e plantas úteis na vida Amazônica. Belém: CIFOR, Imazon, 2005. 304p.

SILVA, J. N. M. Manejo florestal. 3.ed. Belém: Embrapa Amazônia Oriental/Brasília: Embrapa Informação Tecnológica, 2001. 49p.

SILVA, J. N. M. et al. Growth and yield of a tropical rain forest in the Brazilian Amazon 13 years after logging. Forest Ecology and Management, v.71, n.3, p.267-274, 1995.

SMITH, J. et al. Cobertura florestal secundária em pequenas propriedades rurais na Amazônia: implicações para a agricultura de corte e queima. Belém: Embrapa Amazônia Oriental, 2000. 43p. (Documento, 51)

SOUZA, D. R.; SOUZA, A. L. Emprego do método BDq de seleção após a exploração florestal em floresta ombrófila densa de terra firme, Amazônia Oriental. Revista Árvore, v.29, n.4, p.617-625, 2005.

VIDAL, E. et al. Manejo de cipós para a redução do impacto da exploração madeireira na Amazônia Oriental. In: VIDAL, E.; GERWING, J. J.

Ecologia e manejo de cipós na

Amazônia Oriental. Belém: Imazon, 2003. p.13-24.

VIDAL, E.; VIANA, V. M.; BATISTA, J. L. F. Crescimento de floresta tropical três anos após colheita de madeira com e sem manejo florestal na Amazônia Oriental. Scientia Forestalis, v.61, p.133-143, 2002. 